\title{
Publisher Correction: Shared heritability and functional enrichment across six solid cancers
}

Xia Jiang et al."

Correction to: Nature Communications https://doi.org/10.1038/s41467-018-08054-4, published online 25 January 2019.

The original version of this Article contained an error in the author affiliations.

Affiliation 46 incorrectly read 'Fondazione Policlinico Universitario A. Gemelli IRCCS, 00168 Roma, Italy.'

This has now been corrected in both the PDF and HTML versions of the Article.

Published online: 23 September 2019

(i) Open Access This article is licensed under a Creative Commons Attribution 4.0 International License, which permits use, sharing, adaptation, distribution and reproduction in any medium or format, as long as you give appropriate credit to the original author(s) and the source, provide a link to the Creative Commons license, and indicate if changes were made. The images or other third party material in this article are included in the article's Creative Commons license, unless indicated otherwise in a credit line to the material. If material is not included in the article's Creative Commons license and your intended use is not permitted by statutory regulation or exceeds the permitted use, you will need to obtain permission directly from the copyright holder. To view a copy of this license, visit http://creativecommons.org/licenses/by/4.0/.

(C) The Author(s) 2019

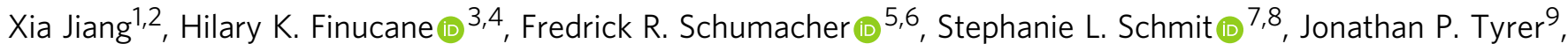
Younghun Han ${ }^{10}$, Kyriaki Michailidou (10 11,12, Corina Lesseur ${ }^{13,14}$, Karoline B. Kuchenbaecker (1) 15,16, Joe Dennis (1) ${ }^{11}$, David V. Conti ${ }^{17}$, Graham Casey ${ }^{18,19}$, Mia M. Gaudet ${ }^{20}$, Jeroen R. Huyghe (iD) Demetrius Albanes ${ }^{22}$, Melinda C. Aldrich ${ }^{23}$, Angeline S. Andrew24, Irene L. Andrulis (1) 25,26, Hoda Anton-Culver ${ }^{27}$, Antonis C. Antoniou11, Natalia N. Antonenkova ${ }^{28}$, Susanne M. Arnold (10 ${ }^{29}$, Kristan J. Aronson 30, Banu K. Arun ${ }^{31}$, Elisa V. Bandera ${ }^{32}$, Rosa B. Barkardottir 33,34, Daniel R. Barnes (1) ${ }^{11}$ Jyotsna Batra (15) 35, Matthias W. Beckmann 37 , Javier Benitez ${ }^{38,39}$, Sara Benlloch ${ }^{11,40}$, Andrew Berchuck ${ }^{41}$, Sonja I. Berndt 22 , Heike Bickeböller ${ }^{42}$, Stephanie A. Bien21,43, Carl Blomqvist ${ }^{44,45}$, Stefania Boccia 46,47 , Natalia V. Bogdanova 28,48,49, Stig E. Bojesen (10 50,51,52, Manjeet K. Bolla11, Hiltrud Brauch (1) 53,54,55, Hermann Brenner 55,56,57, James D. Brenton (1D ${ }^{58}$, Mark N. Brook (1D ${ }^{40}$, Joan Brunet (1) ${ }^{59}$, Hans Brunnström (10) ${ }^{60,61}$, Daniel D. Buchanan (102,63,64, Barbara Burwinkel ${ }^{65,66}$, Ralf Butzow ${ }^{67}$, Gabriella Cadoni ${ }^{46,47}$, Trinidad Caldés ${ }^{68}$, Maria A. Caligo ${ }^{69}$, lan Campbell (10) 70,71 , Peter T. Campbell20, Géraldine Cancel-Tassin (10 ${ }^{72,73,}$ Lisa Cannon-Albright ${ }^{74,75}$, Daniele Campa ${ }^{76,77}$, Neil Caporaso ${ }^{22}$, André L. Carvalho ${ }^{78,79,}$ 
Andrew T. Chan (10) ${ }^{80,81}$, Jenny Chang-Claude ${ }^{76,82}$, Stephen J. Chanock (1) ${ }^{22}$, Chu Chen ${ }^{83}$, David C. Christiani ${ }^{3}$, Kathleen B.M. Claes $\mathbb{1 0}^{84}$, Frank Claessens ${ }^{85}$, Judith Clements ${ }^{35,36}$, J. Margriet Collée ${ }^{86}$, Marcia Cruz Correa ${ }^{87}$, Fergus J. Couch ${ }^{88}$, Angela Cox ${ }^{89}$, Julie M. Cunningham (10 ${ }^{88}$, Cezary Cybulski ${ }^{90}$, Kamila Czene ${ }^{91}$, Mary B. Daly ${ }^{92}$, Anna deFazio 93,94, Peter Devilee (1) 95,96, Orland Diez ${ }^{97}$, Manuela Gago-Dominguez ${ }^{98,99}$, Jenny L. Donovan ${ }^{100}$, Thilo Dörk ${ }^{49}$, Eric J. Duell ${ }^{101}$, Alison M. Dunning ${ }^{9}$, Miriam Dwek ${ }^{102}$, Diana M. Eccles ${ }^{103}$, Christopher K. Edlund ${ }^{104}$, Digna R. Velez Edwards ${ }^{105}$, Carolina Ellberg (10 ${ }^{106}$, D. Gareth Evans ${ }^{107}$, Peter A. Fasching (1) 37,108, Robert L. Ferris ${ }^{109}$, Triantafillos Liloglou (1110, Jane C. Figueiredo ${ }^{111,112,}$ Olivia Fletcher (1) ${ }^{113}$, Renée T. Fortner ${ }^{76}$, Florentia Fostira ${ }^{114}$, Silvia Franceschi ${ }^{115}$, Eitan Friedman 116,117, Steven J. Gallinger ${ }^{118,119,120}$, Patricia A. Ganz ${ }^{121}$, Judy Garber ${ }^{122}$, José A. García-Sáenz (B) ${ }^{68}$, Simon A. Gayther 123,124,125, Graham G. Giles ${ }^{126,127,128}$, Andrew K. Godwin ${ }^{129}$, Mark S. Goldberg ${ }^{130,131}$, David E. Goldgar ${ }^{132}$, Ellen L. Goode ${ }^{133}$, Marc T. Goodman ${ }^{134,135}$, Gary Goodman ${ }^{136}$, Kjell Grankvist (1) ${ }^{137}$, Mark H. Greene (10 ${ }^{138}$, Henrik Gronberg (1) ${ }^{91}$, Jacek Gronwald ${ }^{90}$, Pascal Guénel (1) ${ }^{139}$, Niclas Håkansson ${ }^{140}$, Per Hall91,141, Ute Hamann ${ }^{142}$, Freddie C. Hamdy ${ }^{143}$, Robert J. Hamilton ${ }^{144}$, Jochen Hampe (1) ${ }^{145}$, Aage Haugen $^{146}$, Florian Heitz ${ }^{147,148}$, Rolando Herrero ${ }^{149}$, Peter Hillemanns ${ }^{49}$, Michael Hoffmeister ${ }^{56}$, Estrid Høgdall150,151, Yun-Chul Hong ${ }^{152}$, John L. Hopper ${ }^{127}$, Richard Houlston (1) 153, Peter J. Hulick (1) 154,155, David J. Hunter ${ }^{1}$, David G. Huntsman ${ }^{156,157,158}$, Gregory Idos ${ }^{17}$, Evgeny N. Imyanitov ${ }^{159}$, Sue Ann Ingles ${ }^{17}$, Claudine Isaacs ${ }^{160}$, Anna Jakubowska90,161, Paul James (10) ${ }^{71,162}$, Mark A. Jenkins (1) ${ }^{62,127}$, Mattias Johansson ${ }^{14}$, Mikael Johansson ${ }^{163}$, Esther M. John ${ }^{164}$, Amit D. Joshi (10) ${ }^{3,165}$, Radka Kaneva ${ }^{166}$, Beth Y. Karlan ${ }^{167}$, Linda E. Kelemen ${ }^{168}$, Tabea Kühl ${ }^{169}$, Kay-Tee Khaw ${ }^{170}$, Elza Khusnutdinova ${ }^{171,172}$, Adam S. Kibel ${ }^{173}$, Lambertus A. Kiemeney ${ }^{174}$, Jeri Kim75, Susanne K. Kjaer ${ }^{150,176}$, Julia A. Knight ${ }^{177,178}$, Manolis Kogevinas $39,179,180,181$, Zsofia Kote-Jarai ${ }^{40}$, Stella Koutros ${ }^{182}$, Vessela N. Kristensen ${ }^{183,184,185}$, Jolanta Kupryjanczyk ${ }^{186}$, Martin Lacko ${ }^{187}$, Stephan Lam ${ }^{188}$, Diether Lambrechts (189,190, Maria Teresa Landi ${ }^{191}$, Philip Lazarus ${ }^{192}$, Nhu D. Le ${ }^{193}$, Eunjung Lee ${ }^{123}$, Flavio Lejbkowicz ${ }^{194}$, Heinz-Josef Lenz ${ }^{104}$, Goska Leslie (iD ${ }^{11}$, Davor Lessel (iD) ${ }^{195}$, Jenny Lester ${ }^{167}$, Douglas A. Levine (i) ${ }^{196,197}$, Li Li ${ }^{198,199}$, Christopher I. Li200, Annika Lindblom ${ }^{201}$, Noralane M. Lindor ${ }^{202}$, Geoffrey Liu ${ }^{203}$, Fotios Loupakis ${ }^{204}$, Jan Lubiński90, Lovise Maehle205, Christiane Maier206, Arto Mannermaa207,208,209, Loic Le Marchand210, Sara Margolin ${ }^{211}$, Taymaa May 212, Lesley McGuffog ${ }^{11}$, Alfons Meindl213, Pooja Middha76,214, Austin Miller (1) ${ }^{215}$, Roger L. Milne 126,127, Robert J. Maclnnis ${ }^{126,127}$, Francesmary Modugno 216,217, Marco Montagna ${ }^{218}$, Victor Moreno (10) ${ }^{219}$, Kirsten B. Moysich 220, Lorelei Mucci ${ }^{3}$, Kenneth Muir (10) 221,222, Anna Marie Mulligan223,224, Katherine L. Nathanson (1) ${ }^{225}$, David E. Neal ${ }^{58,143,226}$, Andrew R. Ness ${ }^{227}$, Susan L. Neuhausen ${ }^{228}$, Heli Nevanlinna (10 229, Polly A. Newcomb (10) 21,43, Lisa F. Newcomb 21,230, Finn Cilius Nielsen ${ }^{231}$,

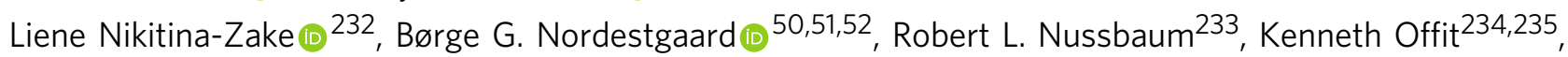
Edith Olah236, Ali Amin Al Olama (1) 11,237, Olufunmilayo I. Olopade (i] ${ }^{238}$, Andrew F. Olshan 239,240, Håkan Olsson 106, Ana Osorio 38,39, Hardev Pandha241, Jong Y. Park ${ }^{242}$, Nora Pashayan (10) 243,244, Michael T. Parsons (10 245, Tanja Pejovic 246,247, Kathryn L. Penney ${ }^{81}$, Wilbert H.M. Peters ${ }^{248}$, Catherine M. Phelan242, Amanda I. Phipps 21,249, Dijana Plaseska-Karanfilska (1) 250, Miranda Pring 251, Darya Prokofyeva ${ }^{171}$, Paolo Radice ${ }^{252}$, Kari Stefansson ${ }^{253}$, Susan J. Ramus ${ }^{254,255}$, Leon Raskin (D) ${ }^{256}$, Gad Rennert (10 257, Hedy S. Rennert ${ }^{257}$, Elizabeth J. van Rensburg ${ }^{258}$, Marjorie J. Riggan ${ }^{41}$, Harvey A. Risch ${ }^{259}$, Angela Risch (10) 260,261,262, Monique J. Roobol (10) 263, Barry S. Rosenstein264,265, Mary Anne Rossing83,266, Kim De Ruyck ${ }^{267}$, Emmanouil Saloustros (1) ${ }^{268}$, Dale P. Sandler ${ }^{269}$, Elinor J. Sawyer 270 , Matthew B. Schabath (1D 242, Johanna Schleutker (1D) 271,272,273, Marjanka K. Schmidt (10) 274,275 , V. Wendy Setiawan ${ }^{276}$, Hongbing Shen ${ }^{277}$, Erin M. Siegel ${ }^{7}$, Weiva Sieh ${ }^{278}$, Christian F. Singer ${ }^{279}$, 


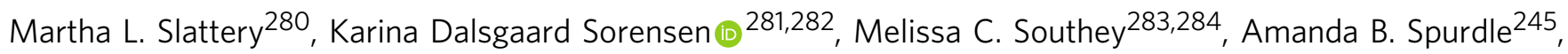
Janet L. Stanford ${ }^{21,249}$, Victoria L. Stevens ${ }^{20}$, Sebastian Stintzing (1D ${ }^{285}$, Jennifer Stone (10 ${ }^{127,286}$, Karin Sundfeldt ${ }^{287}$, Rebecca Sutphen ${ }^{288}$, Anthony J. Swerdlow 40,289, Eloiza H. Tajara290,291, Catherine M. Tangen 292 , Adonina Tardon (10 293, Jack A. Taylor 269,294, M. Dawn Teare ${ }^{295}$, Manuel R. Teixeira (10) 296,297, Mary Beth Terry ${ }^{298}$, Kathryn L. Terry 299,300, Stephen N. Thibodeau ${ }^{88,}$ Mads Thomassen 301, Line Bjørge 302,303, Marc Tischkowitz 304,305, Amanda E. Toland (10 306, Diana Torres ${ }^{142,307,}$ Paul A. Townsend ${ }^{308}$, Ruth C. Travis ${ }^{309}$, Nadine Tung ${ }^{310}$, Shelley S. Tworoger ${ }^{3,242}$, Cornelia M. Ulrich ${ }^{21,311,}$ Nawaid Usmani312,313, Celine M. Vachon ${ }^{133}$, Els Van Nieuwenhuysen ${ }^{314}$, Ana Vega 39,315, Miguel Elías Aguado-Barrera (1) ${ }^{315}$, Qin Wang ${ }^{11}$, Penelope M. Webb (1) ${ }^{316}$, Clarice R. Weinberg ${ }^{317}$, Stephanie Weinstein ${ }^{22}$, Mark C. Weissler ${ }^{318}$, Jeffrey N. Weitze ${ }^{319}$, Catharine M.L. West ${ }^{320}$, Emily White E $^{321,322,}$ Alice S. Whittemore 323,324 , H-Erich Wichmann $325,326,327$, Fredrik Wiklund ${ }^{91}$, Robert Winqvist ${ }^{328,329}$, Alicja Wolk (140,330, Penella Woll ${ }^{331}$, Michael Woods ${ }^{332}$, Anna H. Wu ${ }^{123}$, Xifeng Wu ${ }^{333}$, Drakoulis Yannoukakos (114) Wei Zheng (1) ${ }^{256}$, Shanbeh Zienolddiny ${ }^{146}$, Argyrios Ziogas (i) ${ }^{27}$, Kristin K. Zorn ${ }^{334}$, Jacqueline M. Lane 4,335, Richa Saxena 4,335, Duncan Thomas ${ }^{123}$, Rayjean J. Hung 177,178, Brenda Diergaarde ${ }^{336,337}$, James McKay ${ }^{338}$, Ulrike Peters (10) 249, Li Hsu²1, Montserrat García-Closas ${ }^{22}$, Rosalind A. Eeles (10) 40,339, Georgia Chenevix-Trench ${ }^{245}$, Paul J. Brennan (1) ${ }^{14}$, Christopher A. Haiman ${ }^{17}$, Jacques Simard ${ }^{340,}$ Douglas F. Easton (10) 9,11, Stephen B. Gruber ${ }^{123}$, Paul D.P. Pharoah (10) 9,11, Alkes L. Price (10) 1,3,4, Bogdan Pasaniuc ${ }^{341}$, Christopher I. Amos ${ }^{342}$, Peter Kraft ${ }^{1,3}$ \& Sara Lindström²1,249

${ }^{1}$ Program in Genetic Epidemiology and Statistical Genetics, Harvard T.H. Chan School of Public Health, 677 Huntington Ave, Boston, MA 02115, USA. ${ }^{2}$ Unit of Cardiovascular Epidemiology, Institute of Environmental Medicine, Karolinska Institutet, Nobels vagen 13, 17177 Stockholm, Sweden. ${ }^{3}$ Department of Epidemiology, Harvard T.H. Chan School of Public Health, 677 Huntington Ave, Boston, MA 02115, USA. ${ }^{4}$ Program in Medical and Population Genetics, Broad Institute of MIT and Harvard, 75 Ames St, Cambridge, MA 02142, USA. ${ }^{5}$ Department of Population and Quantitative Health Sciences, Case Western Reserve University, 10900 Eucid Avenue, Cleveland, OH 44106, USA. ${ }^{6}$ Seidman Cancer Center, University Hospitals, Cleveland, OH 44106, USA. 7 Department of Cancer Epidemiology, H. Lee Moffitt Cancer Center and Research Institute, 12902 Magnolia Dr. MRC-CANCONT, Tampa, FL 33612, USA. ${ }^{8}$ Department of Gastrointestinal Oncology, H. Lee Moffitt Cancer Center and Research Institute, 12902 Magnolia Dr. MRC-CANCONT, Tampa, FL 33612, USA. ${ }^{9}$ Centre for Cancer Genetic Epidemiology, Department of Oncology, University of Cambridge, 2 Worts' Causeway, Cambridge CB1 8RN, UK. ${ }^{10}$ Department of Biomedical Data Science, The Geisel School of Medicine at Dartmouth, 1 Medical Center Drive, Lebanon, NH 03756, USA. ${ }^{11}$ Centre for Cancer Genetic Epidemiology, Department of Public Health and Primary Care, University of Cambridge, 2 Worts' Causeway, Cambridge CB1 8RN, UK. ${ }^{12}$ Department of Electron Microscopy/Molecular Pathology, The Cyprus Institute of Neurology and Genetics, 1683 Nicosia, Cyprus. ${ }^{13}$ Genetic Epidemiology Group, International Agency for Research on Cancer, 150 Cours Albert Thomas, 69008 Lyon, France. ${ }^{14}$ Section of Genetics, International Agency for Research on Cancer, 150 cours Albert Thomas, 69008 Lyon, France. ${ }^{15}$ Division of Psychiatry, University College London, Maple House, 149 Tottenham Court Road, London W1T 7NF, UK. ${ }^{16}$ UCL Genetics Institute, University College London, Gower Street, London WC1E 6BT, UK. ${ }^{17}$ Department of Preventive Medicine, Keck School of Medicine, University of Southern California Norris Comprehensive Cancer Center, Los Angeles, CA 48109, USA. ${ }^{18}$ Public Health Sciences, University of Virginia, P.O. Box 800717 Charlottesville, VI 22908, USA. ${ }^{19}$ Center for Public Health Genomics, University of Virginia, P.O. Box 800717 Charlottesville, VI 22908, USA. ${ }^{20}$ Epidemiology Research Program, American Cancer Society, 250 Williams Street NW, Atlanta, GA 30303, USA. ${ }^{21}$ Public Health Sciences Division, Fred Hutchinson Cancer Research Center, 1100 Fairview Ave. N., Seattle, WA 98109-1024, USA. ${ }^{22}$ Division of Cancer Epidemiology and Genetics, National Cancer Institute, 9609 Medical Center Dr, Rockville, MD 20850, USA. ${ }^{23}$ Department of Thoracic Surgery, Division of Epidemiology, Vanderbilt University Medical Center, 609 Oxford House, Nashville, TN 37232, USA. ${ }^{24}$ Department of Neurology, Dartmouth-Hitchcock Medical Center, 7927 Rubin Building, Room 860, One Medical Center Drive, Lebanon, NH 3756, USA. ${ }^{25}$ Fred ALitwin Center for Cancer Genetics, Lunenfeld-Tanenbaum Research Institute of Mount Sinai Hospital, 600 University Avenue, Toronto, ON M5G1X5, Canada. ${ }^{26}$ Department of Molecular Genetics, University of Toronto, 1 King's College Circle, Toronto, ON M5S1A8, Canada. ${ }^{27}$ Department of Epidemiology, Genetic Epidemiology Research Institute, University of California Irvine, 224 Irvine Hall, Irvine, CA 92617, USA.

${ }^{28}$ NNAlexandrov Research Institute of Oncology and Medical Radiology, Settlement of Lesnoy-2, 223040 Minsk, Belarus. ${ }^{29}$ Markey Cancer Center, University of Kentucky, 800 Rose Street, cc445, Lexington, KY 40508, USA. ${ }^{30}$ Department of Public Health Sciences, and Cancer Research Institute, Queen's University, 10 Stuart Street, Kingston, ON K7L 3N6, Canada. ${ }^{31}$ Department of Breast Medical Oncology, University of Texas MD Anderson Cancer Center, 1155 Pressler St, Houston, TX 77030, USA. ${ }^{32}$ Cancer Prevention and Control Program, Rutgers Cancer Institute of New Jersey, 195 Little Albany Street, Room 5568, New Brunswick, NJ 08903, USA. ${ }^{33}$ Department of Pathology, Landspitali University Hospital, Hringbraut, Reykjavik 101, Iceland. ${ }^{34}$ BMC (Biomedical Centre), Faculty of Medicine, University of Iceland, Vatnsmyrarvegi 16, Reykjavik 101, Iceland. ${ }^{35}$ Australian Prostate Cancer Research Centre-QId, Translational Research Institute, 37 Kent St, Woolloongabba, QLD 4102, Australia. ${ }^{36}$ Institute of Health and Biomedical Innovation and School of Biomedical Science, Queensland University of Technology, 60 Musk Ave, Kelvin Grove, QLD 4059 , Australia. ${ }^{37}$ Department of Gynecology and Obstetrics, Comprehensive Cancer Center Erlangen Nuremberg, University Hospital Erlangen, FriedrichAlexander-University Erlangen-Nuremberg, Universitaetsstrasse 21-23, 91054 Erlangen, Germany. ${ }^{38}$ Human Cancer Genetics Programme, Spanish National Cancer Research Centre (CNIO), Calle de Melchor Fernández Almagro, 3, 28029 Madrid, Spain. ${ }^{39}$ Biomedical Network on Rare Diseases (CIBERER), AvMonforte de Lemos, 3-5Pabellón 11Planta 0, 28029 Madrid, Spain. ${ }^{40}$ Division of Genetics and Epidemiology, The Institute of Cancer 
Research, 15 Cotswold Road, London SM2 5NG, UK. ${ }^{41}$ Department of Obstetrics and Gynecology, Duke University Medical Center, 25171 Morris Bldg, Durham, NC 27710, USA. ${ }^{42}$ Department of Genetic Epidemiology, University Medical Center Goettingen, Humboldtallee 32, 37073 Goettingen, Germany. ${ }^{43}$ School of Public Health, University of Washington, 1959 NE Pacific Street, Health Science Buidling, F-350, Seattle, WA 98195, USA. ${ }^{44}$ Department of Oncology, Helsinki University Hospital, University of Helsinki, Haartmaninkatu 4, 00290 Helsinki, Finland. ${ }^{45}$ Department of Oncology, Örebro University Hospital, 70185 Örebro, Sweden. ${ }^{46}$ Fondazione Policlinico Universitario A. Gemelli IRCCS, 00168 Roma, Italy. ${ }^{47}$ Università Cattolica del Sacro Cuore, 00168 Roma, Italy. ${ }^{48}$ Department of Radiation Oncology, Hannover Medical School, CarlNeuberg-Straße 1, 30625 Hannover, Germany. ${ }^{49}$ Gynaecology Research Unit, Hannover Medical School, Carl-Neuberg-Straße 1, 30625 Hannover, Germany. ${ }^{50}$ Copenhagen General Population Study, Herlev and Gentofte Hospital, Copenhagen University Hospital, Herlev Ringvej 75, 2730 Herlev, Denmark. ${ }^{51}$ Department of Clinical Biochemistry, Herlev and Gentofte Hospital, Copenhagen University Hospital, Herlev Ringvej 75, 2730 Herlev, Denmark. ${ }^{52}$ Faculty of Health and Medical Sciences, University of Copenhagen, Blegdamsvej 3B, 2200 Copenhagen, Denmark. ${ }^{53}$ DrMargarete Fischer-Bosch-Institute of Clinical Pharmacology, Auerbachstr112, 70376 Stuttgart, Germany. ${ }^{54}$ University of Tübingen, Geschwister-Scholl-Platz, 72074 Tübingen, Germany. ${ }^{55}$ German Cancer Consortium (DKTK), German Cancer Research Center (DKFZ), Im Neuenheimer Feld 280,69120 Heidelberg, Germany. ${ }^{56}$ Division of Clinical Epidemiology and Aging Research, German Cancer Research Center (DKFZ), Im Neuenheimer Feld 280, 69120 Heidelberg, Germany. ${ }^{57}$ Division of Preventive Oncology, German Cancer Research Center (DKFZ) and National Center for Tumor Diseases (NCT), Im Neuenheimer Feld 280, 69120 Heidelberg, Germany. ${ }^{58}$ Cancer Research UK Cambridge Institute, University of Cambridge, Li Ka Shing Centre, Robinson Way, CB2 ORE Cambridge, UK. ${ }^{59}$ Genetic Counseling Unit, Hereditary Cancer Program, IDIBGI (Institut d'Investigació Biomèdica de Girona), Catalan Institute of Oncology, CIBERONC, AvFrança s/n, 17007 Girona, Spain. ${ }^{60}$ Clinical Sciences, Lund University, Box 117,22100 Lund, Sweden. ${ }^{61}$ Department of Genetics and Pathology, Division of Laboratory Medicine, 22185 Lund, Sweden. ${ }^{62}$ University of Melbourne Centre for Cancer Research, Victorian Comprehensive Cancer Centre, Parkville, VIC 3010, Australia. ${ }^{63}$ Colorectal Oncogenomics Group, Department of Clinical Pathology, The University of Melbourne, Parkville, VIC 3010, Australia. ${ }^{64}$ Genomic Medicine and Family Cancer Clinic, Royal Melbourne Hospital, Parkville, VIC 3010, Australia. ${ }^{65}$ Department of Obstetrics and Gynecology, University of Heidelberg, Im Neuenheimer Feld 440,69120 Heidelberg, Germany. ${ }^{66}$ Molecular Epidemiology Group, C080, German Cancer Research Center (DKFZ), Im Neuenheimer Feld 280,69120 Heidelberg, Germany. ${ }^{67}$ Department of Pathology, University of Helsinki and Helsinki University Hospital, Biomedicum Helsinki 4th Floor, Haartmaninkatu 8, 00029 Helsinki, Finland. ${ }^{68}$ Medical Oncology Department, Hospital Clínico San Carlos, Instituto de Investigación Sanitaria San Carlos (IdISSC), Centro Investigación Biomédica en Red de Cáncer (CIBERONC), Calle del Prof Martín Lagos, 28040 Madrid, Spain. ${ }^{69}$ Section of Genetic Oncology, Department of Laboratory Medicine, University and University Hospital of Pisa, via Roma 67, 56126 Pisa, Italy. ${ }^{70}$ Peter MacCallum Cancer Center, 305 Grattan Street, Melbourne, VIC 3000, Australia. ${ }^{71}$ Sir Peter MacCallum Department of Oncology, The University of Melbourne, 305 Grattan Street, Melbourne, VIC 3000, Australia. ${ }^{72}$ Sorbonne Université, GRC No5 ONCOTYPE-URO, Tenon Hospital, 75020 Paris, France. ${ }^{73} \mathrm{CeRePP}$, Tenon Hospital, 75020 Paris, France. ${ }^{74}$ Division of Genetic Epidemiology, Department of Medicine, University of Utah School of Medicine, Salt Lake City, UT 84112, USA. ${ }^{75}$ George EWahlen Department of Veterans Affairs Medical Center, Salt Lake City, UT 84112 , USA. ${ }^{76}$ Division of Cancer Epidemiology, German Cancer Research Center (DKFZ), Im Neuenheimer Feld 280, 69120 Heidelberg, Germany. ${ }^{77}$ Department of Biology, University of Pisa, 56126 Pisa, Italy. ${ }^{78}$ Molecular Oncology Research Center, Barretos Cancer Hospital, Rua Antenor Duarte Villela, 1331, Barretos, SP 784-400, Brazil. ${ }^{79}$ Head and Neck Surgery Department, Barretos Cancer Hospital, Pio XII, 1331, Antenor Duarte Villela St, Barretos, SP 14784-400, Brazil. ${ }^{80}$ Division of Gastroenterology, Massachusetts General Hospital, 55 Fruit Street, Boston, MA 02114, USA. ${ }^{81}$ Channing Division of Network Medicine, Department of Medicine, Brigham and Women's Hospital, Harvard Medical School, 181 Longwood Avenue, Boston, MA 02115, USA. ${ }^{82}$ Cancer Epidemiology Group, University Cancer Center Hamburg (UCCH), University Medical Center HamburgEppendorf, Martinistraße 52, 20246 Hamburg, Germany. ${ }^{83}$ Program in Epidemiology, Division of Public Health Sciences, Fred Hutchinson Cancer Research Center, 1100 Fairview Ave N, Seattle, WA 98109, USA. ${ }^{84}$ Centre for Medical Genetics, Ghent University, De Pintelaan 185, 9000 Gent, Belgium. ${ }^{85}$ Molecular Endocrinology Laboratory, Department of Cellular and Molecular Medicine, KU Leuven, Leuven 3000, Belgium. ${ }^{86}$ Department of Clinical Genetics, Erasmus University Medical Center, Wytemaweg 80, 3015 Rotterdam, CN, The Netherlands. ${ }^{87}$ University of Puerto Rico Medical Sciences Campus and Comprehensive Cancer Center, San Juan, PR 00936, USA. ${ }^{88}$ Department of Laboratory Medicine and Pathology, Mayo Clinic, 200 First StSW, Rochester, MN 55905, USA. ${ }^{89}$ Sheffield Institute for Nucleic Acids (SInFoNiA), Department of Oncology and Metabolism, University of Sheffield, Western Bank, Sheffield S10 2TN, UK. ${ }^{90}$ International Hereditary Cancer Center, Department of Genetics and Pathology, Pomeranian Medical University, ulUnii Lubelskiej 1, 71-252 Szczecin, Poland. ${ }^{91}$ Department of Medical Epidemiology and Biostatistics, Karolinska Institutet, Karolinska Univ Hospital, 17176 Stockholm, Sweden. ${ }^{92}$ Department of Clinical Genetics, Fox Chase Cancer Center, 333 Cottman Ave, Philadelphia, PA 19111, USA. ${ }^{93}$ Centre for Cancer Research, The Westmead Institute for Medical Research, The University of Sydney, 176 Hawkesbury Rd, Sydney, NSW 2145, Australia. ${ }^{94}$ Department of Gynaecological Oncology, Westmead Hospital, Hawkesbury Rd \& Darcy Rd, Sydney, NSW 2145, Australia. ${ }^{95}$ Department of Pathology, Leiden University Medical Center, Albinusdreef 2, 2333 ZA Leiden, The Netherlands. ${ }^{96}$ Department of Human Genetics, Leiden University Medical Center, Albinusdreef 2, 2333 ZA Leiden, The Netherlands. ${ }^{97}$ Oncogenetics Group, Clinical and Molecular Genetics Area, Vall d'Hebron Institute of Oncology (VHIO), University Hospital, Vall d'Hebron, Passeig de la Vall d'Hebron 119-129, 08035 Barcelona, Spain. ${ }^{98}$ Genomic Medicine Group, Galician Foundation of Genomic Medicine, Instituto de Investigación Sanitaria de Santiago de Compostela (IDIS), Complejo Hospitalario Universitario de Santiago, SERGAS, Travesía da Choupana S/N, 15706 Santiago de Compostela, Spain. ${ }^{99}$ Moores Cancer Center, University of California, San Diego, 3855 Health Sciences Drive, La Jolla, CA 92037, USA. ${ }^{100}$ School of Social and Community Medicine, University of Bristol, Bristol BS8 1TH, UK. ${ }^{101}$ Unit of Nutrition and Cancer, Cancer Epidemiology Research Program, Catalan Institute of Oncology (ICO-IDIBELL), AvGran Via 199-203, L'Hospitalet de Llobregat, 08908 Barcelona, Spain. ${ }^{102}$ Department of Biomedical Sciences, Faculty of Science and Technology, University of Westminster, 309 Regent Street, London W1B 2HW, UK. ${ }^{103}$ Cancer Sciences Academic Unit, Faculty of Medicine, University of Southampton, Tremona Road, Southampton SO16 6YD, UK. ${ }^{104}$ Department of Medicine, Keck School of Medicine, University of Southern California, Los Angeles, CA 90033, USA. ${ }^{105}$ Vanderbilt Epidemiology Center, Vanderbilt Genetics Institute, Department of Obstetrics and Gynecology, Vanderbilt University Medical Center, 2525 West End Avenue, Suite 600, Nashville, TN 37203, USA. ${ }^{106}$ Department of Cancer Epidemiology, Clinical Sciences, Lund University, Barngatan 4, Skånes universitetssjukhus, 22242 Lund, Sweden. ${ }^{107}$ Manchester Centre for Genomic Medicine, Division of Evolution and Genomic Sciences, University of Manchester, St Mary's Hospital, Central Manchester University Hospitals NHS Foundation Trust, Oxford Road, Manchester M13 9WL, UK. ${ }^{108}$ David Geffen School of Medicine, Department of Medicine Division of Hematology and Oncology, University of California at Los Angeles, 10833 Le Conte Ave, Los Angeles, CA 90095, USA. ${ }^{109}$ Department of Otolaryngology, UPMC Hillman Cancer Center, Cancer Pavilion, University of Pittsburgh, Suite 500, 5150 Centre Avenue, Pittsburgh, PA 15232, USA. ${ }^{10}$ Molecular and Clinical Cancer Medicine, Roy Castle Lung Cancer Research Programme, The University of Liverpool Institute of Translational Medicine, The Wiliam Duncan Building, 6 West Derby Street, Liverpool L69 3BX, UK. ${ }^{111}$ Samuel Oschin Comprehensive Cancer Institute, Cedars-Sinai Medical Center, 8700 Beverly Boulevard, Los Angeles, CA 90048, USA. ${ }^{12}$ Keck School of Medicine, 
University of Southern California, 1450 Biggy Street, Los Angeles, CA 90033, USA. ${ }^{113}$ The Breast Cancer Now Toby Robins Research Centre, The Institute of Cancer Research, 123 Old Brompton Road, London SW7 3RP, UK. ${ }^{114}$ Molecular Diagnostics Laboratory, INRASTES, National Centre for Scientific Research 'Demokritos', Neapoleos 10, AgParaskevi, Athens 15310, Greece. ${ }^{115}$ Section of Infections, International Agency for Research on Cancer, 150 cours Albert Thomas, 69008 Lyon, France. ${ }^{116}$ The Susanne Levy Gertner Oncogenetics Unit, Chaim Sheba Medical Center, Emek HaEla St 1, 52621 Ramat Gan, Israel. ${ }^{117}$ Sackler Faculty of Medicine, Tel Aviv University, Haim Levanon 30, 69978 Ramat Aviv, Israel. ${ }^{118}$ Department of Surgery, Mount Sinai Hospital, 600 University Avenue, Toronto, ON M5G 1X5, Canada. ${ }^{119}$ Samuel Lunenfeld Research Institute, 600 University Avenue, Toronto, ON M5G 1X5, Canada. ${ }^{120}$ University Health Network Toronto General Hospital, 200 Elizabeth St, Toronto, ON M5G 2C4, Canada. ${ }^{121}$ Schools of Medicine and Public Health, Division of Cancer Prevention \& Control Research, Jonsson Comprehensive Cancer Centre, UCLA, 650 Charles Young Drive South, Los Angeles, CA 90095-6900, USA. ${ }^{122}$ Cancer Risk and Prevention Clinic, Dana-Farber Cancer Institute, 450 Brookline Avenue, Boston, MA 02215, USA. ${ }^{123}$ Department of Preventive Medicine, Keck School of Medicine, University of Southern California, 1975 Zonal Ave, Los Angeles, CA 90033, USA. ${ }^{124}$ Center for Cancer Prevention and Translational Genomics, Samuel Oschin Comprehensive Cancer Institute, Cedars-Sinai Medical Center, Spielberg Building, 8725 Alden Dr, Los Angeles, CA 90048, USA. ${ }^{125}$ Department of Biomedical Sciences, Cedars-Sinai Medical Center, Spielberg Building, 8725 Alden Dr, Los Angeles, CA 90048, USA. ${ }^{126}$ Cancer Epidemiology \& Intelligence Division, Cancer Council Victoria, 615 St Kilda Road, Melbourne, VIC 3004, Australia. ${ }^{127}$ Centre for Epidemiology and Biostatistics, Melbourne School of Population and Global Health, The University of Melbourne, Level 1, 723 Swanston Street, Melbourne, VIC 3010, Australia. ${ }^{128}$ Department of Epidemiology and Preventive Medicine, Monash University, Melbourne, VIC, Australia. ${ }^{129}$ Department of Pathology and Laboratory Medicine, University of Kansas Medical Center, 3901 Rainbow Blvd, Kansas City, KS 66160, USA. ${ }^{130}$ Department of Medicine, McGill University, 1001 Decarie Boulevard, Montréal, QC H4A3J1, Canada. ${ }^{131}$ Division of Clinical Epidemiology, Royal Victoria Hospital, McGill University, 1001 Decarie Boulevard, Montréal, QC H4A3J1, Canada. ${ }^{132}$ Department of Dermatology, Huntsman Cancer Institute, University of Utah School of Medicine, 2000 Circle of Hope, Salt Lake City, UT 84112, USA. ${ }^{133}$ Department of Health Sciences Research, Mayo Clinic, 200 First StSW, Rochester, MN 55905, USA. ${ }^{134}$ Cancer Prevention and Control, Samuel Oschin Comprehensive Cancer Institute, Cedars-Sinai Medical Center, 8700 Beverly Blvd, Room 1S37, Los Angeles, CA 90048, USA. ${ }^{135}$ Community and Population Health Research Institute, Department of Biomedical Sciences, Cedars-Sinai Medical Center, 8700 Beverly Blvd, Room 1S37, Los Angeles, CA 90048, USA. ${ }^{136}$ Public Health Sciences Division, Swedish Cancer Institute, 1221 Madison StSte 300, Seattle, WA 98109, USA. ${ }^{137}$ Unit of Clinical Chemistry, Department of Medical Biosciences, Umeå University, By 6M van 2,

Sjukhusomradet, Umea universitet, 90185 Umea, Sweden. ${ }^{138}$ Clinical Genetics Branch, National Cancer Institute, DCEG, 9609 Medical Center Dr, Bethesda, MD 20850-9772, USA. ${ }^{139}$ Cancer \& Environment Group, Center for Research in Epidemiology and Population Health (CESP), INSERM, University Paris-Sud, University Paris-Saclay, 94805 Villejuif, France. ${ }^{140}$ Department of Environmental Medicine, Division of Nutritional Epidemiology, Karolinska Institutet, Nobels väg 13, SE-171 77, SE-171 Stockholm, Sweden. ${ }^{141}$ Department of Oncology, Södersjukhuset, Sjukhusbacken 10, 11883 Stockholm, Sweden. ${ }^{142}$ Molecular Genetics of Breast Cancer, German Cancer Research Center (DKFZ), Im Neuenheimer Feld 580, 69120 Heidelberg, Germany. ${ }^{143}$ Nuffield Department of Surgical Sciences, Faculty of Medical Science, John Radcliffe Hospital, University of Oxford, Oxford OX1 2JD, UK. ${ }^{144}$ Department of Surgical Oncology, Princess Margaret Cancer Centre, 610 University Avenue, Toronto, Ontario M5G2M9, Canada. ${ }^{145}$ Department of Internal Medicine 1, University Hospital Dresden, Technische Universität Dresden (TU Dresden), 01307 Dresden, Germany. ${ }^{146}$ National Institute of Occupational Health (STAMI), Gydas vei 8, 0033 Oslo, Norway. ${ }^{147}$ Department of Gynecology and Gynecologic Oncology, DrHorst Schmidt Kliniken Wiesbaden, Ludwig-Erhard-Straße 100, 65199 Wiesbaden, Germany. ${ }^{148}$ Department of Gynecology and Gynecologic Oncology, Kliniken Essen-Mitte/EvangHuyssens-Stiftung/Knappschaft GmbH, Henricistrasse 92,45136 Essen, Germany. ${ }^{149}$ Early Detection and Prevention Section, International Agency for Research on Cancer, 150 cours Albert Thomas, 69008 Lyon, France. ${ }^{150}$ Department of Virus, Lifestyle and Genes, Danish Cancer Society Research Center, Strandboulevarden 49, DK-2100 Copenhagen, Denmark.

${ }^{151}$ Molecular Unit, Department of Pathology, Herlev Hospital, University of Copenhagen, Herlev Ringvej 75, DK-2730 Herlev, Denmark. ${ }^{152}$ Preventive Medicine, Seoul National University College of Medicine, 1 Gwanak-ro, Gwanak-gu, Seoul 151 742, Korea. ${ }^{153}$ German Research Center for Environmental Health, Institute for Cancer Research, Ingolstadter Landstr1, London SM2 5NG, UK. ${ }^{154}$ Center for Medical Genetics, NorthShore University HealthSystem, 1000 Central St, Evanston, IL 60201, USA. ${ }^{155}$ The University of Chicago Pritzker School of Medicine, 924 E 57th St, Chicago, IL 60637, USA. ${ }^{156}$ British Columbia's Ovarian Cancer Research (OVCARE) Program, Vancouver General Hospital, BC Cancer Agency and University of British Columbia, \#3427-600 West 10th Avenue, Vancouver, BC V5Z 4E6, Canada. ${ }^{157}$ Department of Molecular Oncology, BC Cancer Agency Research Centre, \#3427-600 West 10th Avenue, Vancouver, BC V5Z 4E6, Canada. ${ }^{158}$ Department of Pathology and Laboratory Medicine, University of British Columbia, \#3427-600 West 10th Avenue, Vancouver, BC V5Z 4E6, Canada. ${ }^{159}$ NNPetrov Institute of Oncology, Leningradskaya ul, 68, StPetersburg, Russia 197758. ${ }^{160}$ Lombardi Comprehensive Cancer Center, Georgetown University, 3800 Reservoir Road, Washington, DC 20007, USA. ${ }^{161}$ Independent Laboratory of Molecular Biology and Genetic Diagnostics, Pomeranian Medical University, Rybacka 1, 70-204 Szczecin, Poland. ${ }^{162}$ Parkville Familial Cancer Centre, Peter MacCallum Cancer Center, 305 Grattan Street, Melbourne, VIC 3000, Australia. ${ }^{163}$ Department of Radiation Sciences, Umeå University, By 6M van 2, Sjukhusomradet, Umea universitet, 90185 Umea, Sweden. ${ }^{164}$ Department of Medicine, Division of Oncology and Stanford Cancer Institute, Stanford University School of Medicine, 780 Welch Rd, Stanford, CA 94304, USA. ${ }^{165}$ Clinical and Translational Epidemiology Unit, Massachusetts General Hospital, 02114 Boston, MA, USA. ${ }^{166}$ Molecular Medicine Center, Department of Medical Chemistry and Biochemistry, Medical Faculty, Medical University of Sofia, Sofia 1504, Bulgaria. ${ }^{167}$ Women's Cancer Program at the Samuel Oschin Comprehensive Cancer Institute, Cedars-Sinai Medical Center, 8700 Beverly Boulevard, Los Angeles, CA 90048 , USA. ${ }^{168}$ Hollings Cancer Center and Department of Public Health Sciences, Medical University of South Carolina, 68 President Street Bioengineering Building, MSC955, Charleston, SC 29425, USA. ${ }^{169}$ Cancer Epidemiology, University Cancer Center Hamburg (UCCH), University Medical Center Hamburg-Eppendorf, Martinistraße 52, 20246 Hamburg, Germany. ${ }^{170}$ Clinical Gerontology, Department of Public Health and Primary Care, University of Cambridge, 2 Worts' Causeway, Cambridge CB1 8RN, UK. ${ }^{171}$ Department of Genetics and Fundamental Medicine, Bashkir State University, ulZaki Validi 32, Ufa, Russia 450076. ${ }^{172}$ Institute of Biochemistry and Genetics, Ufa Scientific Center of Russian Academy of Sciences, 71 prosp Oktyabrya, Ufa, Russia 450054. ${ }^{173}$ Division of Urologic Surgery, Brigham and Womens Hospital, Boston, Massachusettes 02115, USA. ${ }^{174}$ Radboud Institute for Health Sciences, Radboud University Medical Center, Geert Grooteplein 21, 6525 EZ Nijmegen, The Netherlands. ${ }^{175}$ Department of Genitourinary Medical Oncology, University of Texas MD Anderson Cancer Center, 1155 Pressler St, Houston, TX 77030, USA. ${ }^{176}$ Department of Gynaecology, Rigshospitalet, University of Copenhagen, Blegdamsvej 9, DK-2100 Copenhagen, Denmark.

${ }^{177}$ Prosserman Centre for Population Health Research, Lunenfeld-Tanenbaum Research Institute, Sinai Health System, 60 Murray Street, Toronto, Ontario M5T 3L9, Canada. ${ }^{178}$ Division of Epidemiology, Dalla Lana School of Public Health, University of Toronto, 155 College Street, Toronto, ON M5T3M7, Canada. ${ }^{179}$ Centre for Research in Environmental Epidemiology (CREAL), ISGlobal, 08036 Barcelona, Spain. ${ }^{180}$ IMIM (Hospital del Mar Research Institute), Barcelona 08003, Spain. ${ }^{181}$ Universitat Pompeu Fabra (UPF), Barcelona 08002, Spain. ${ }^{182}$ Division of Cancer Epidemiology and Genetics, National Cancer Institute, Department of Health and Human Services, National Institutes of Health, 9609 Medical Center Dr, Bethesda, 
MD 20892, USA. ${ }^{183}$ Department of Cancer Genetics, Institute for Cancer Research, Oslo University Hospital Radiumhospitalet, Ullernchausseen 70, 0379 Oslo, Norway. ${ }^{184}$ Faculty of Medicine, Institute of Clinical Medicine, University of Oslo, Kirkeveien 166, 0450 Oslo, Norway. ${ }^{185}$ Department of Clinical Molecular Biology, Oslo University Hospital, University of Oslo, Kirkeveien 166, 0450 Oslo, Norway. ${ }^{186}$ Department of Pathology and Laboratory Diagnostics, the Maria Sklodowska-Curie Institute - Oncology Center, Roentgena 5, 02-781 Warsaw, Poland. ${ }^{187}$ Head and Neck Surgery, Department of Otorhinolaryngology, Maastricht University Medical Center, PDebyelaan 25, POBox 5800, 6202 AZ Maastricht, The Netherlands. ${ }^{188}$ Department of Integrative Oncology, British Columbia Cancer Agency, Room 10-111 675 West 10th Avenue, Vancouver, BC V5Z1L3, Canada. ${ }^{189}$ VIB Center for Cancer Biology, VIB, Herestraat 49, 3001 Leuven, Belgium. ${ }^{190}$ Laboratory for Translational Genetics, Department of Human Genetics, University of Leuven, Oude Markt 13, 3000 Leuven, Belgium. ${ }^{191}$ Integrative Tumor Epidemiology Branch, DCEG, National Cancer Institute, 9609 Medical Center Drive, Room SG/7E106, Rockville, MD 20850, USA. ${ }^{192}$ College of Pharmacy, Washington State University, PBS 431 PO Box 1495, Spokane, WA 99210-1495, USA. ${ }^{193}$ Cancer Control Research, BC Cancer Agency, 675 West 10th Avenue, Vancouver, BC V5Z 1L3, Canada. ${ }^{194}$ Clalit Health Services, Clalit National Israeli Cancer Control Center, Carmel Medical Center, 2 Horev Street, 3436212 Haifa, Israel. ${ }^{195}$ Institute of Human Genetics, University Medical Center Hamburg-Eppendorf, Martinistraße 52, 20246 Hamburg, Germany. ${ }^{196}$ Gynecology Service,

Department of Surgery, Memorial Sloan Kettering Cancer Center, 1275 York Avenue, New York, NY 10065, USA. ${ }^{197}$ Gynecologic Oncology, Laura and Isaac Pearlmutter Cancer Center, NYU Langone Medical Center, 240 East 38th Street 19th Floor, New York, NY 10016, USA. ${ }^{198}$ Department of Family Medicine and Community Health, Mary Ann Swetland Center for Environmental Health, Case Western Reserve University, Cleveland, OH 44106, USA. ${ }^{199}$ Servicio Galego de Saude (SERGAS), Instituto de Investigación Sanitaria de Santiago de Compostela (IDIS), 15706 Santiago De Compostela, Spain. ${ }^{200}$ Translational Research Program, Fred Hutchinson Cancer Research Center, Seattle, WA 98109, USA. ${ }^{201}$ Department of Molecular Medicine and Surgery, Karolinska Institutet, Karolinska Univ Hospital, 17176 Stockholm, Sweden. ${ }^{202}$ Health Sciences Research, Mayo Clinic Arizona, 13400 EShea Blvd, Scottsdale, AZ 85259, USA. ${ }^{203}$ Epidemiology Division, Princess Margaret Cancer Centre, 610 University Avenue, Toronto, ON M5G2M9, Canada. ${ }^{204}$ Unit of Oncology 1, Department of Clinical and Experimental Oncology, Istituto Oncologico Veneto IRCCS, 35122 Padua, Italy. ${ }^{205}$ Department of Medical Genetics, Oslo University Hospital, Kirkeveien 166, 0450 Oslo, Norway. ${ }^{206}$ Institute of Human Genetics, University Hospital Ulm, Prittwitzstrasse 43, 89075 Ulm, Germany. ${ }^{207}$ Translational Cancer Research Area, University of Eastern Finland, Yliopistonranta 1, 70210 Kuopio, Finland. ${ }^{208}$ Institute of Clinical Medicine, Pathology and Forensic Medicine, University of Eastern Finland, KuopioYliopistonranta 1, 70210, Finland. ${ }^{209}$ Imaging Center, Department of Clinical Pathology, Kuopio University Hospital, Puijonlaaksontie 2, 70210 Kuopio, Finland. ${ }^{210}$ Epidemiology Program, University of Hawaii Cancer Center, 701 Ilalo St, Honolulu, HI 96813, USA. ${ }^{211}$ Department of Clinical Science and Education, Södersjukhuset, Karolinska Institutet, Stockholm 17177, Sweden. ${ }^{212}$ Division of Gynecologic Oncology, University Health Network, Princess Margaret Hospital, 610 University Avenue, OPG Wing, 6-811, Toronto, ON M5G 2M9, Canada. ${ }^{213}$ Division of Gynaecology and Obstetrics, , Technische Universität München, Arcisstraße 21, 80333 Munich, Germany. ${ }^{214}$ Faculty of Medicine, University of Heidelberg, In Neuenheimer Feld 672, 69120 Heidelberg, Germany. ${ }^{215}$ NRG Oncology, Statistics and Data Management Center, Roswell Park Cancer Institute, Elm \& Carlton Streets, Buffalo, NY 14263, USA. ${ }^{216}$ Womens Cancer Research Center, Magee-Womens Research Institute and Hillman Cancer Center, Pittsburgh, PA 15213, USA. ${ }^{217}$ Division of Gynecologic Oncology, Department of Obstetrics, Gynecology and Reproductive Sciences, University of Pittsburgh School of Medicine, 300 Halket Street, Pittsburgh, PA 15213, USA. ${ }^{218}$ Immunology and Molecular Oncology Unit, Veneto Institute of Oncology IOV - IRCCS, Via Gattamelata 64, Padua 35128, Italy. ${ }^{219}$ Catalan Institute of Oncology, Bellvitge Biomedical Research Institute (IDIBELL), Consortium for Biomedical Research in Epidemiology and Public Health (CIBERESP) and University of Barcelona, Barcelona 08908, Spain. ${ }^{220}$ Division of Cancer Prevention and Control, Roswell Park Cancer Institute, Elm \& Carlton Streets, Buffalo, NY 14263, USA. ${ }^{221}$ Division of Population Health, Health Services Research and Primary Care, University of Manchester, Oxford Road, Manchester M13 9PL, UK. ${ }^{222}$ Division of Health Sciences, Warwick Medical School, University of Warwick, Coventry CV4 7AL, UK. ${ }^{223}$ Department of Laboratory Medicine and Pathobiology, University of Toronto, 1 King's College Circle, Toronto, ON M5S1A8, Canada. ${ }^{224}$ Laboratory Medicine Program, University Health Network, 200 Elizabeth Street, Toronto, ON M5G2C4, Canada. ${ }^{225}$ Department of Medicine, Abramson Cancer Center, Perelman School of Medicine at the University of Pennsylvania, 3400 Civic Center Boulevard, Philadelphia, PA 19104, USA. ${ }^{226}$ Department of Oncology, Addenbrooke's Hospital, University of Cambridge, Cambridge CB1 8RN, UK. ${ }^{227}$ NIHR Bristol Biomedical Research Centre Nutrition Theme, University of Bristol, Upper Maudlin Street, Bristol BS2 8AE, UK. ${ }^{228}$ Department of Population Sciences, Beckman Research Institute of City of Hope, 1500 E Duarte, Duarte, CA 91010, USA. ${ }^{229}$ Department of Obstetrics and Gynecology, Helsinki University Hospital, University of Helsinki, Haartmaninkatu 8 , 00290 Helsinki, Finland. ${ }^{230}$ Department of Urology, University of Washington, Seattle, Washington 98195, USA. ${ }^{231}$ Center for Genomic Medicine, Rigshospitalet, Copenhagen University Hospital, Blegdamsvej 9, DK-2100 Copenhagen, Denmark. ${ }^{232}$ Latvian Biomedical Research and Study Centre, Ratsupites str 1, Riga LV-1067, Latvia. ${ }^{233}$ Cancer Genetics and Prevention Program, University of California San Francisco, 1600 Divisadero St, San Francisco, CA 94143-1714, USA. ${ }^{234}$ Clinical Genetics Research Lab, Department of Cancer Biology and Genetics, Memorial Sloan-Kettering Cancer Center, 1275 York Avenue, New York, NY 10065, USA. ${ }^{235}$ Clinical Genetics Service, Department of Medicine, Memorial Sloan-Kettering Cancer Center, 1275 York Avenue, New York, NY 10065, USA. ${ }^{236}$ Department of Molecular Genetics, National Institute of Oncology, Ráth György u7-9, 1122 Budapest, Hungary. ${ }^{237}$ Department of Clinical Neurosciences, University of Cambridge, Cambridge CB2 OQQ, UK. ${ }^{238}$ Center for Clinical Cancer Genetics, The University of Chicago, 5841S Maryland Ave, Chicago, IL 60637, USA. ${ }^{239}$ Department of Epidemiology, Gillings School of Global Public Health, University of North Carolina, 135 Dauer Dr, Chapel Hill, NC 27599-7435, USA. ${ }^{240}$ UNC Lineberger Comprehensive Cancer Center, 450 West Dr, Chapell Hill, NC 27599, USA. ${ }^{241}$ The University of Surrey, Guildford, Surrey GU2 7XH, UK. ${ }^{242}$ Department of Cancer Epidemiology, HLee Moffitt Cancer Center and Research Institute, 12902 Magnolia Drive, Tampa, FL 33612, USA. ${ }^{243}$ Department of Applied Health Research, University College London, 1-19 Torrington Place, London WC1E 6BT, UK. ${ }^{244}$ Centre for Cancer Genetic Epidemiology, Department of Oncology, Strangeways Laboratory, University of Cambridge, Cambridge CB1 8RN, UK. ${ }^{245}$ Department of Genetics and Computational Biology, QIMR Berghofer Medical Research Institute, 300 Herston Road, Brisbane, QLD 4006, Australia. ${ }^{246}$ Department of Obstetrics and Gynecology, Oregon Health \& Science University, 3181 SW Sam Jackson Park Road, L-466, Portland, OR 97239, USA. ${ }^{247}$ Knight Cancer Institute, Oregon Health \& Science University, 3181 SW Sam Jackson Park Road, L-466, Portland, OR 97239, USA. ${ }^{248}$ Department of Gastroenterology, Radboud University Nijmegen Medical Center, Geert Grooteplein Zuid 10, Internal BOBox 433, 6525 GA Nijmegen, The Netherlands. ${ }^{249}$ Department of Epidemiology, University of Washington School of Public Health, 1959 NE Pacific St, Seattle, WA 98195, USA. ${ }^{250}$ Research Centre for Genetic Engineering and Biotechnology 'Georgi DEfremov', Macedonian Academy of Sciences and Arts, Boulevard Krste Petkov Misirkov, 1000 Skopje, Republic of Macedonia. ${ }^{251}$ Bristol Dental School, University of Bristol, Lower Maudlin Street, Bristol BS1 2LY, UK. ${ }^{252}$ Unit of Molecular Bases of Genetic Risk and Genetic Testing, Department of Research, Fondazione IRCCS (Istituto Di Ricovero e Cura a Carattere Scientifico) Istituto Nazionale dei Tumori (INT), Via Giacomo Venezian 1, 20133 Milan, Italy. ${ }^{253}$ Decode genetics, Sturlugata 8, IS-101 ReykjavikReykjaviklceland, Iceland. ${ }^{254}$ School of Women's and Children's Health, Faculty of Medicine, University of NSW Sydney, 18 High St, Sydney, NSW 2052, Australia. ${ }^{255}$ The Kinghorn Cancer Centre, Garvan Institute of Medical Research, 384 Victoria Street, Sydney, NSW 2010, Australia. ${ }^{256}$ Division of Epidemiology, Department 
of Medicine, Vanderbilt Epidemiology Center, Vanderbilt-Ingram Cancer Center, Vanderbilt University School of Medicine, $116121 s t$ Ave S \# D3300, Nashville, TN 37232, USA. ${ }^{257}$ Clalit National Cancer Control Center, Carmel Medical Center and Technion Faculty of Medicine, 7 Michal Street, 34362 Haifa, Israel. ${ }^{258}$ Department of Genetics, University of Pretoria, Private Bag X323, Arcadia 0007, South Africa. ${ }^{259}$ Department of Chronic Disease Epidemiology, Yale School of Public Health, 60 College St, New Haven, CT 06510, USA. ${ }^{260}$ Cancer Center Cluster Salzburg at PLUS, Department of Molecular Biology, University of Salzburg, Billrothstr11, 5020 Salzburg, Austria. ${ }^{261}$ Division of Epigenomics and Cancer Risk Factors, DKFZ - German Cancer Research Center, Im Neuenheimer Feld 280, 69120 Heidelberg, Germany. ${ }^{262}$ Member of the German Center for Lung Research (DZL), Translational Lung Research Center Heidelberg (TLRC-H), 69120 Heidelberg, Germany. ${ }^{263}$ Department of Urology, Erasmus University Medical Center, Wytemaweg 80, 3015 CN Rotterdam, The Netherlands. ${ }^{264}$ Department of Radiation Oncology, Icahn School of Medicine at Mount Sinai, 1425 Madison Avenue, New York, NY 10029, USA. ${ }^{265}$ Department of Genetics and Genomic Sciences, Icahn School of Medicine at Mount Sinai, 1425 Madison Avenue, New York, NY 10029, USA. ${ }^{266}$ Department of Epidemiology, University of Washington, M4 C308, 1100 Fairview Ave N, Seattle, WA 98109, USA. ${ }^{267}$ Faculty of Medicine and Health Sciences, Basic Medical Sciences, Ghent University, De Pintelaan 185, 9000 Gent, Belgium. ${ }^{268}$ Hereditary Cancer Clinic, University Hospital of Heraklion, Voutes, 71110 Heraklion, Greece. ${ }^{269}$ Epidemiology Branch, National Institute of Environmental Health Sciences, NIH, 111TWAlexander Drive, Research Triangle Park, NC 27709, USA. ${ }^{270}$ Research Oncology, Guy's Hospital, King's College London, Guy's Hospital Great Maze Pond, London SE1 9RT, UK. ${ }^{271}$ Institute of Biomedicine, University of Turku, 20014 Turku, Finland. ${ }^{272}$ Division of Laboratory, Department of Medical Genetics, Turku University Hospital, 20014 Turku, Finland. ${ }^{273}$ Prostate Cancer Research Center, Faculty of Medicine and Life Sciences and BioMediTech Institute, University of Tampere, 33014 Tampere, Finland. ${ }^{274}$ Division of Molecular Pathology, The Netherlands Cancer Institute - Antoni van Leeuwenhoek Hospital, Plesmanlaan 121, 1066 CX Amsterdam, The Netherlands. ${ }^{275}$ Division of Psychosocial Research and Epidemiology, The Netherlands Cancer Institute - Antoni van Leeuwenhoek hospital, Plesmanlaan 121, 1066 CX Amsterdam, The Netherlands. ${ }^{276}$ Department of Preventive Medicine, Keck School of Medicine, University of Southern California, 1450 Biggy Street, Los Angeles, CA 90033, USA. ${ }^{277}$ Department of Epidemiology and Biostatistics, Jiangsu Key Lab of Cancer Biomarkers, Prevention and Treatment, Collaborative Innovation Center for Cancer Personalized Medicine, School of Public Health, Nanjing Medical University, 101 Longmian Ave, Jiangning District, 211166 Nanjing, People's Republic of China. ${ }^{278}$ Department of Genetics and Genomic Sciences, Department of Population Health Science and Policy, Icahn School of Medicine at Mount Sinai, 1425 Madison Avenue, 2nd floor, New York, NY 10029, USA. ${ }^{279}$ Dept of OB/GYN and Comprehensive Cancer Center, Medical University of Vienna, Waehringer Guertel 18-20, 1090 Vienna, Austria. ${ }^{280}$ Department of Internal Medicine, University of Utah Health Sciences Center, 295 Chipeta Way, Salt Lake City, UT 84132, USA. ${ }^{281}$ Department of Molecular Medicine, Aarhus University Hospital, DK-8200 Aarhus, Denmark. ${ }^{282}$ Department of Clinical Medicine, Aarhus University, DK-8200 Aarhus, Denmark. ${ }^{283}$ Precision Medicine, School of Clinical Sciences at Monash Health, Monash University, 246 Clayton Road, Clayton, VIC 3168, Australia. ${ }^{284}$ Department of Clinical Pathology, The University of Melbourne, Cnr Grattan Street and Royal Parade, Melbourne, VIC 3010, Australia. ${ }^{285}$ Department of Medicine III, University Hospital, LMU Munich, Marchioninistr15, 81377 Munich, Germany. ${ }^{286}$ The Curtin UWA Centre for Genetic Origins of Health and Disease, Curtin University and University of Western Australia, 35 Stirling Hwy, Perth, WA 6000, Australia. ${ }^{287}$ Department of Obstetrics and Gynecology, Sahlgrenska Cancer Center, Inst Clinical Scienses, University of Gothenburg, Blå stråket 6, 41345 Gothenburg, Sweden. ${ }^{288}$ Epidemiology Center, College of Medicine, University of South Florida, 3650 Spectrum Blvd, Suite 100, Tampa, FL 33612, USA. ${ }^{289}$ Division of Breast Cancer Research, The Institute of Cancer Research, London SW7 3RP, UK. ${ }^{290}$ Department of Molecular Biology, School of Medicine of São José do Rio Preto, Av Brig Faria Lima 5416 Vila São Pedro, São José do Rio Preto, SP 15090-000, Brazil. ${ }^{291}$ Department of Genetics and Evolutive Biology, Institute of Biosciences, University of São Paulo, Rua do Matão, 321, São Paulo, SP 05508090, Brazil. ${ }^{292}$ SWOG Statistical Center, Fred Hutchinson Cancer Research Center, Seattle, Washington 98109, USA. ${ }^{293}$ Faculty of Medicine, University of Oviedo and CIBERESP, Campus del Cristo s/n, 33006 Oviedo, Spain. ${ }^{294}$ Epigenetic and Stem Cell Biology Laboratory, National Institute of Environmental Health Sciences, NIH, 111TWAlexander Drive, Research Triangle Park, NC 27709, USA. ${ }^{295}$ Medical Statistics Group, School of Health and Related Research (ScHARR), University of Sheffield, Regent Court, 30 Regent Street, Sheffield S1 4DA, UK. ${ }^{296}$ Department of Genetics, Portuguese Oncology Institute, Rua DrAntónio Bernardino de Almeida 62, 4220-072 Porto, Portugal. ${ }^{297}$ Biomedical Sciences Institute (ICBAS), University of Porto, RJorge de Viterbo Ferreira 228, 4050-013 Porto, Portugal. ${ }^{298}$ Department of Epidemiology, Mailman School of Public Health, Columbia University, 722 West 168th Street, New York, NY 10032, USA. ${ }^{299}$ Obstetrics and Gynecology Epidemiology Center, Brigham and Women's Hospital, 221 Longwood Avenue RFB 368, Boston, MA 02115, USA. ${ }^{300}$ Harvard THChan School of Public Health, 221 Longwood Avenue RFB 368, Boston, MA 02115, USA. ${ }^{301}$ Department of Clinical Genetics, Odense University Hospital, Sonder Boulevard 29, 5000 Odence C, Denmark. ${ }^{302}$ Department of Gynecology and Obstetrics, Haukeland University Hospital, 5021 Bergen, Norway. ${ }^{303}$ Centre for Cancer Biomarkers CCBIO, Department of Clinical Science, University of Bergen, 5021 Bergen, Norway. ${ }^{304}$ Program in Cancer Genetics, Departments of Human Genetics and Oncology, McGill University, 1001 Decarie Boulevard, Montréal, QC H4A3J1, Canada. ${ }^{305}$ Department of Medical Genetics, Cambridge University, Hills Road, Cambridge CB2 OQQ, UK. ${ }^{306}$ Department of Cancer Biology and Genetics, The Ohio State University, 460W12th Avenue, Columbus, OH 43210, USA. ${ }^{307}$ Institute of Human Genetics, Pontificia Universidad Javeriana, Carrera 7 No40-90, Bogota, Colombia. ${ }^{308}$ Division of Cancer Sciences, Manchester Cancer Research Centre, Faculty of Biology, Medicine and Health, Manchester Academic Health Science Centre, NIHR Manchester Biomedical Research Centre, Health Innovation Manchester, University of Manchester, Manchester M20 4GJ, UK. ${ }^{309}$ Cancer Epidemiology Unit, Nuffield Department of Population Health, University of Oxford, Oxford OX3 7LF, UK. ${ }^{310}$ Department of Medical Oncology, Beth Israel Deaconess Medical Center, 330 Brookline Avenue, Boston, MA 02215, USA. ${ }^{311}$ Huntsman Cancer Institute and Department of Population Health Sciences, University of Utah, 2000 Circle of Hope, Rm 4125, Salt Lake City, UT 84112, USA. ${ }^{312}$ Department of Oncology, Cross Cancer Institute, University of Alberta, 116 St \& 85 Ave, Edmonton AB T6G 2R3, Canada. ${ }^{313}$ Division of Radiation Oncology, Cross Cancer Institute, University of Alberta, 116 St \& 85 Ave, Edmonton AB T6G 2R3, Canada. ${ }^{314}$ Division of Gynecologic Oncology, Department of Obstetrics and Gynaecology and Leuven Cancer Institute, University Hospitals Leuven, Herestraat 49, 3000 Leuven, Belgium. ${ }^{315}$ Fundación Pública Galega Medicina Xenómica \& Instituto de Investigación Sanitaria de Santiago de Compostela, calle Choupana s/n, 15706 Santiago De Compostela, Spain. ${ }^{316}$ Population Health Department, QIMR Berghofer Medical Research Institute, 300 Herston Road, Brisbane, QLD 4006, Australia. ${ }^{317}$ Biostatistics and Computational Biology Branch, National Institute of Environmental Health Sciences, NIH, 111TWAlexander Drive, Research Triangle Park, NC 27709, USA. ${ }^{318}$ Department of Otolaryngology/Head and Neck Surgery, University of North Carolina at Chapel Hill, Chapel Hill 27514 NC, USA. ${ }^{319}$ City of Hope Clinical Cancer Genomics Community Research Network, 1500 East Duarte Road, Duarte, CA 91010, USA. ${ }^{320}$ Division of Cancer Sciences, University of Manchester, Manchester Cancer Research Centre, Manchester Academic Health Science Centre,, The Christie Hospital NHS Foundation Trust, Manchester M13 9PL, UK. ${ }^{321}$ Fred Hutchinson Cancer Research Center, 1100 Fairview Ave N, Seattle, WA 98109, USA.

${ }^{322}$ Department of Epidemiology, University of Washington, 1100 Fairview Ave N, Seattle, WA 98109, USA. ${ }^{323}$ Department of Health Research and Policy - Epidemiology, Stanford University School of Medicine, 259 Campus Drive, Stanford, CA 94305, USA. ${ }^{324}$ Department of Biomedical Data Science, Stanford University School of Medicine, 259 Campus Drive, Stanford, CA 94305, USA. ${ }^{325}$ Institute of Medical Informatics, Biometry and 
Epidemiology, Chair of Epidemiology, Ludwig Maximilians University, Neuherberg D-85764, Munich 803539 Bavaria, Germany. ${ }^{326}$ Helmholtz Zentrum Munchen, German Research Center for Environmental Health (GmbH), Institute of Epidemiology, Ingolstadter Landstr1, 85764 Neuherberg, Germany. ${ }^{327}$ Institute of Medical Statistics and Epidemiology, Technical University Munich, Munich 80333, Germany. ${ }^{328}$ Laboratory of Cancer Genetics and Tumor Biology, Cancer and Translational Medicine Research Unit, Biocenter Oulu, University of Oulu, Aapistie 5A, 90220 Oulu, Finland. ${ }^{329}$ Laboratory of Cancer Genetics and Tumor Biology, Northern Finland Laboratory Centre Oulu, Aapistie 5A, 90220 Oulu, Finland. ${ }^{330}$ Department of Surgical Sciences, Uppsala University, 75185 Uppsala, Sweden. ${ }^{331}$ Academic Unit of Clinical Oncology, University of Sheffield, Weston Park Hospital, Whitham Road, Sheffield S10 2SJ, UK. ${ }^{332}$ Discipline of Genetics, Memorial University of Newfoundland, StJohn's, NL A1C 5S7, Canada. ${ }^{333}$ Department of Epidemiology, Division of Cancer Prevention and Population Science, The University of Texas MD Anderson Cancer Center, 1515 Holcombe Blvd, Houston, TX 77030, USA. ${ }^{334}$ Magee-Womens Hospital, University of Pittsburgh School of Medicine, 300 Halket St, Pittsburgh, PA 15213, USA. ${ }^{335}$ Center for Genomic Medicine and Department of Anasthesia, Massachusetts General Hospital, Boston, MA 02114, USA. ${ }^{336}$ Human Genetics, Graduate School of Public Health, University of Pittsburgh, UPMC Cancer Pavilion, Suite 4C, Office \# 467, 5150 Centre Avenue, Pittsburgh, PA 15232, USA. ${ }^{337}$ UPMC Hillman Cancer Center, Pittsburgh 15232 PA, USA. ${ }^{338}$ Genetic Cancer Susceptibility Group, International Agency for Research on Cancer, 150 cours Albert Thomas, 69008 Lyon, France. ${ }^{339}$ Oncogenetics Team, The Institute of Cancer Research and Royal Marsden NHS Foundation Trust, Downs Road, Sutton SM2 5NG, UK. ${ }^{340}$ Genomics Center, Centre Hospitalier Universitaire de Québec - Université Laval Research Center, 2705 Laurier Boulevard, Québec City, QC G1V4G2, Canada. ${ }^{341}$ UCLA Path and Lab Med, University of California, 10833 Le Conte Ave, Los Angeles, CA 190095, USA. ${ }^{342}$ Department of Medicine, Epidemiology Section, Institute for Clinical and Translational Research, Baylor Medical College, One Baylor Plaza, MS: BCM451, Suite 100D, Houston, TX 77030-3411, USA 